\title{
ROBUST ALGORITHM FOR DIGITIZATION OF DEGRADED ELECTROCARDIOGRAM PAPER RECORDS
}

\author{
Rupali Patil ${ }^{\mathbf{1}}$ and R. G. Karandikar ${ }^{2}$ \\ ${ }^{I}$ Department of Electronics and Telecommunication Engineering, Rajiv Gandhi Institute of Technology, Mumbai, India \\ ${ }^{2}$ Department of Electronics and Telecommunication Engineering, K.J. Somaiya College of Engineering, India
}

\begin{abstract}
Electrocardiogram (ECG) paper records are used commonly for diagnosing heart abnormalities. The stored ECG paper records are recorded on thermal paper and may face ink evaporation problem over the time. Generally, to overcome this problem ECG paper record is scanned and stored as an image. However, the addition of noise during scanning such as low resolution scan, blurring, folding of paper, nonuniform lighting, orientation etc. can create difficulty in information retrieval. Current work robustly handles various degradation problems encountered in ECG paper scanning using modified $k$-fill algorithm. The proposed algorithm is tested with 836 ECG paper recordings with different types of degradations like aging effect, folding effect, ink evaporation effect, blurring effect and low resolution effect. We extracted clinically important parameters such as heart rate etc. with accuracy of $97.33 \%$ and abnormalities such as bradycardia, tachycardia, and atrial flutter from the ECG paper records using perceptual spectral centroid method. Overall accuracy of our prediction algorithm was found out to be $\mathbf{9 8 . 6 \%}$. We assume our work would be low cost, preliminary expert mechanism at rural places in the absence of expert cardiologist.
\end{abstract}

\section{Keywords:}

Degraded ECG Records, ECG Prediction, Signal Retrieval and Analysis, Perceptual Spectral Centroid Method, Vertical Scanning Signal Extraction

\section{INTRODUCTION}

An electrocardiogram (ECG) is a test that checks for problems with the electrical activity of the heart. An ECG shows the electrical activity of the heart as line tracings on paper (Fig.1(a)). The spikes and dips in the tracings are called waves or signals. These recorded signals then used for abnormality detection.

As computers are an integral part in the medical field, developing an application for rapid and easy disease diagnosis is essential. 'Digital signals' are a method of effectively using highspeed computational ability and the bulk-volume-data-handling capacity of the computer in ECG data preservation. For ECG preservation, the ECG waveform is printed usually on paper, and the printed ECG is preserved by patients and produced when asked by a doctor. The problem encountered while reading the data from documented ECG paper is the damage caused by paper tearing or the disappearance of the signal traces because of various types of noise deposition. To overcome this problem, the ECG paper record is converted into digital time series signals through the ECG digitization process. Ultimately, electronic conservation of the documented ECG records is crucial.
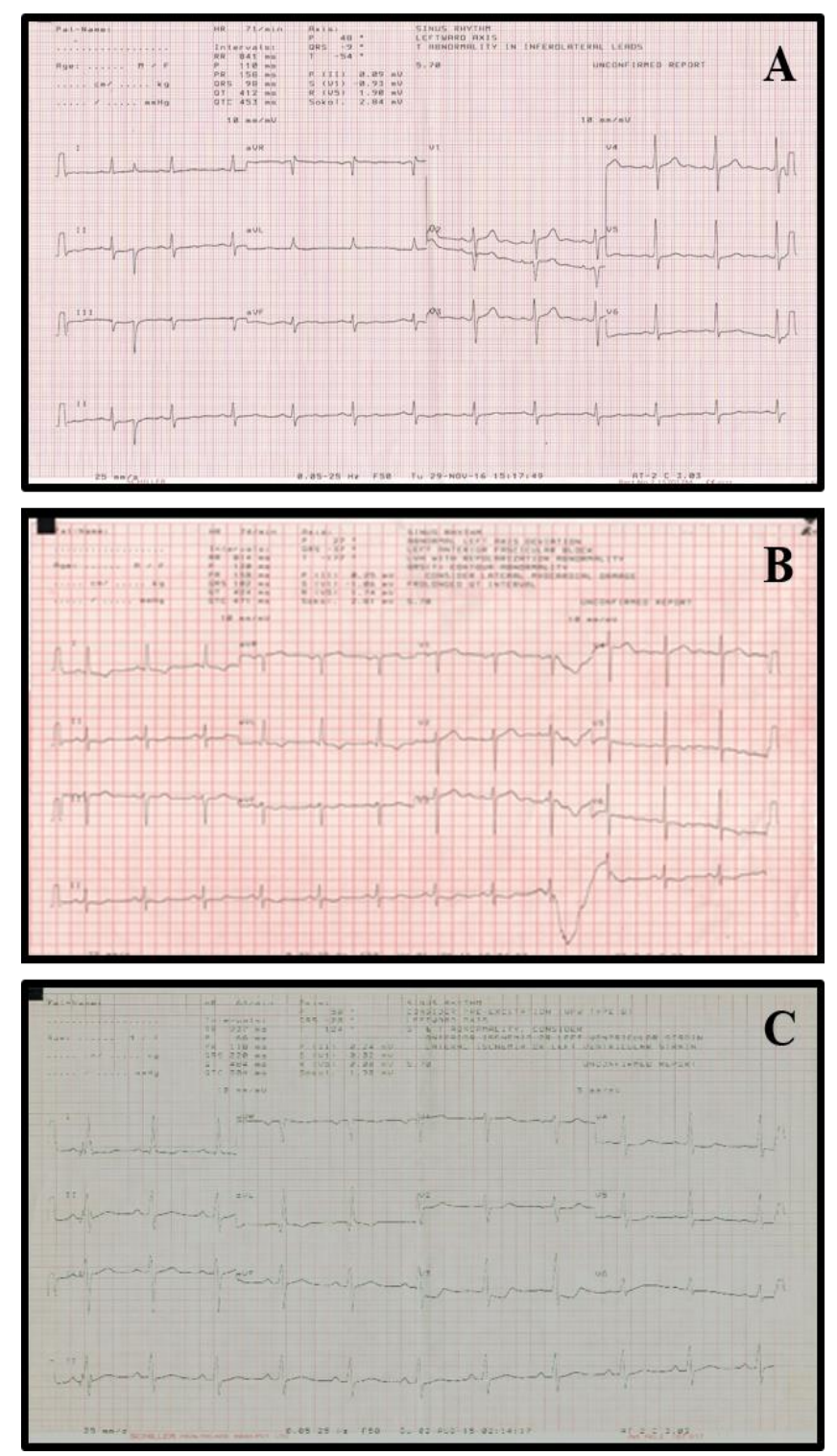

Fig.1. Electrocardiogram paper record with (a) no degradation (b) ink evaporation effect (c) folding effect

Paper ECG records are insubstantial and it is not possible to maintain same quality forever. The problem encountered while digitizing the data from documented ECG paper is the damage caused by ink evaporation (Fig.1(b)), blurring, folding of paper (Fig.1(c)), paper tearing etc. The initial part of this study focuses on eliminating the problems encountered in ECG digitization using k-fill algorithm which is modified using anti-aliasing method. The accuracy of the proposed algorithm is validated by extracting clinically crucial parameters such as heart rate (HR), 
R-peak, S-peak, and RR-interval from digitized signals and comparing them with the pre-diagnosed results. The complexity of the proposed algorithm is reduced by employing vertical scanning, which significantly reduces the number of iterations in the scanning process.

Our work focuses on robust image enhancement technique to extract signal from degraded documented ECG records before further processing them. We have also found that time complexity is reduced by employing vertical scanning instead of traditional horizontal scanning. In this paper we have also attempted to estimate abnormalities using perceptual spectral centroid method (PSCM) from signal extracted from degraded paper ECG records. The normal and abnormal records are predicted using of our prediction algorithm (PSCM) which improves overall accuracy.

The remainder of the paper is organized as follows. Related work is presented in section 2. Section 3 propose methodology for the degraded ECG paper record digitization process. The experimental results are presented in section 4 and discussed in section 5. Section 6 presents the conclusion of this study.

\section{RELATED WORK}

The types of scenarios where noise is introduced in the ECG paper records reported in literature are as follows: The graph grid lines can be the source of noise which interfere with the trace of ECG [1], ECG papers damaged due to dust particles leading to salt and pepper noise [2-3], uneven contrast, interfering strokes [4], background spots, humidity absorbed by paper [5] in different areas, Blurring and broken signal [6].

Grid removal is necessary for retrieving the ECG signal from ECG printouts. Many researchers have reported in literature different methods of grid removal from paper records. These methods are mathematical morphology [7] Hough transform [8] projection of lines $[9,10]$. The signal trace is separated from the background grids using the histogram [11].

Previously Baldilini et al. suggested a method for extraction of the ECG trace from the image [12]. However, this method requires the user to fix anchor points for missing peaks and thus the accuracy is affected as per user input. Shi et al. used ECG waveform extracted from paper and parameters were retrieved using K- means method [13]. Ravichandran et al. extracted ECG signal [14] and interfaced with patient's record using optical character recognition (OCR). Lawson et al. used scanning resolution of $200 \mathrm{dpi}$ and grayscale thresholds were used to separate the ECG trace from the background grid lines [15]. Their low resolution method results in loss of data accuracy and grayscale thresholds results in missing pixels. However, all the above mentioned works address the issue of one-dimensional time series signal from fresh paper records without noise. Signal extraction from non-clear and degraded scanned documents is still a challenge. We have tried to address these issues in our current work. Recently perceptual spectral centroid method proposed by Hermes et al. [16] was used for harmonic centroid of string instruments. We have implemented the same method for ECG signals, as it relates closely.

Recently, Waits et al. [17] reviewed various digitization and conversion algorithms and criticised said algorithms, applications, and standardization efforts. A comprehensive list of each paper-to-digital conversion algorithm was discussed in tabular form along with limitations and corresponding solutions. Important points discussed were methods for digitization of paper ECG records, of paper-to-digital ECG conversion, highpoint possible applications and remaining challenges. Generally scanned paper ECG records are RGB images. For extraction of ECG signal from RGB images it is required to convert them into grayscale images. Rajani et al. [18] proposed use of $\mathrm{R}$ plane for ECG signal extraction from RGB image instead of RGB image to gray scale image conversion.

The input images required from digitization of paper ECG records can be obtained from using either scanner or cell phones. Nowadays paper ECG records are available, where all 12 leads are printed on single paper (Fig.1(a)). For extraction of ECG signal from individual lead, most of the authors' manually crop 12-lead image into single lead image. Automatic cropping of 12lead images is proposed by Fernando et al. [19] which reduces manual errors and also corrected distortions.

\section{METHODOLOGY}

The proposed algorithm is tested on 836 actual ECG recordings of patients having various degradation problems. The dataset is taken with proper permission of the medical practitioner and patient (Saikripa Hospital, Mulund). As shown in the first block of Fig.2 the paper ECGs recorded on thermal paper is scanned using flatbed scanner with 300-dpi resolution and stored in the lossless format. Because these images are scanned from the available records, problems such as blurred signal traces, folding of paper, broken ECG traces and non-uniform background intensity were introduced in the scanned paper ECG images.

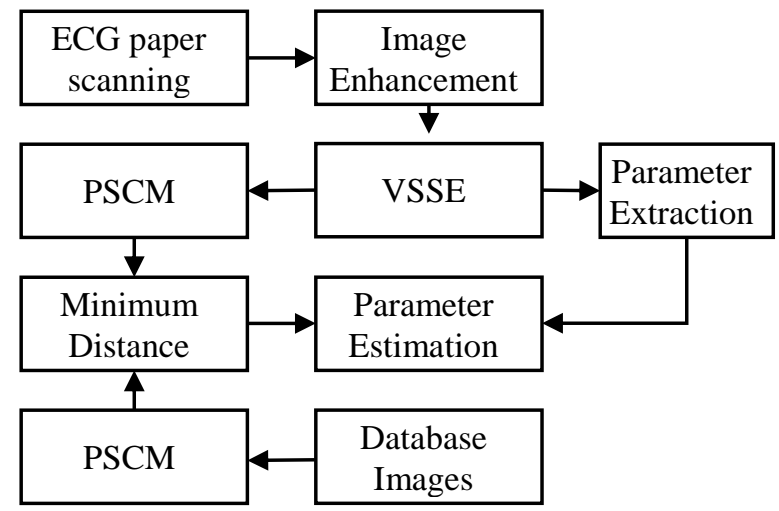

Fig.2. Flow graph of ECG digitization process

Unlike other methods the proposed algorithm is designed specifically for ECG paper digitization without removing the grid of the graph paper. Block 2 is image enhancement. It is the most important step in this algorithm. It involves the steps such as color to gray scale conversion, noise removal, and trace enhancement. Trace enhancement step especially handles the significant problems of blurred and broken old trace using modified antialiasing method (i.e. k-fill algorithm).

The appearance of aliased or jagged diagonal edges, termed "Jaggies", are removed with the method of anti-aliasing. Antialiasing converts aliased edges into smoother edges with higher resolution. The aliased effect occurred due to simply rounding up or down with no intermediate value. This leads to breakage in 
continuities of printed signal trace. This effect generally occurs in old printed documentations specifically in line or curved plots like printed signal or ECG signals. As the archived ECG paper records are degraded or noisy, the noise reduction filter needs to maintain tradeoff between noise reduction and signal retention. The k-Fill filter is utilized for the same that works in two sub iterations by filling white pixels to remove noise and by filling black pixels to maintain signal trace. A $k \times k$ window is moved in raster scan manner over the entire image. The region $(k-2) \times(k-2)$ is called core and the $4(k-1)$ pixels are called the neighbor- hood. The core is filled with either white or black pixel depending on pixel values in the neighbor-hood.

After digital scanning and image enhancement, 2-D image is scanned for high intensity pixels of ECG signal trace. For tracing the signal traversing horizontally, horizontal (row wise) scanning is not suitable as it is difficult to localize the equal gray valued pixels. It requires iterative process for exact localization of such pixels. To reduce this iterative process, vertical scanning signal extraction (VSSE) is used instead of horizontal scanning. In single scan trace (with no iterative process), it can identify the high intensity pixels as there is only one high valued pixel in vertical trace of signal. A 2-dimensional trace matrix stores locations of high valued pixels with reference to the vertical scan line. Signal is plotted using the values of Trace matrix.

For parameter extraction, the very first step required is the identification of axis or base line. The test square pulse in the starting of ECG signal helps to identify the axis. However, in most ECG trace capture procedures, square pulses are absent. After converting 2-D image to 1-D signal, the samples of 1-D signal are stored in vector represented as $[X]=\left[x_{1}, x_{2}, \ldots, x_{n}\right]$. It is found that the most significant and recurring samples usually represent axis of the signal. As the signal is stored as a vector, the axis is calculated by taking mode of $[X]$. The-digitized signal is normalized and the reference line or base line is determined by following formula,

$$
\begin{aligned}
& X=X-\bmod e(X) \\
& X=\frac{X}{\max (a b s(X))}
\end{aligned}
$$

The R-peaks which have maximum and S-peaks have minimum amplitude in the ECG signal are extracted by finding maximum and minimum values of vector $\mathrm{X}$. The number of sample values between consecutive R-peaks is used to find RRinterval and Heart rate (HR) as follows,

$$
\text { HeartRate }=\frac{60}{R R \text { interval }}
$$

After correct extraction of ECG signal in digital form it's parameters and centroid is calculated for diagnosis purpose. PSCM calculates spectral centroid, sampled at frequency with respect to mel-frequency by using 2048 sample window size $(w)$ with $50 \%$ overlap hamming window. Also the spectrogram of the signal is computed and finally a centroid is predicted by converting FFT frequencies to mels.

$$
\operatorname{spectogram}(t, w)=|\operatorname{STFT}(f(t), w)|^{2}
$$

$$
\text { Centroid }=\frac{\sum_{n=0}^{N-1} f(n) x(n)}{\sum_{n-0}^{N-1} x(n)}
$$

Once the centroid is computed for unknown ECG, $f(t)$, it is then classified using minimum distance decoding algorithm. Here the Euclidian distance is computed between the centroid of the unknown sample and centroid of the known disease database sample. Though we got accuracy of $98.6 \%$, still it would be just preliminary scanning mechanism to generate rough estimate of patient condition and not the complete replacement of the expert cardiologist. Further the algorithm can be improved with other matching techniques.

\section{RESULTS}

The algorithm is tested on 836 unprocessed degraded ECG paper recordings unlike online database. The parameters were extracted to validate the proposed algorithm.

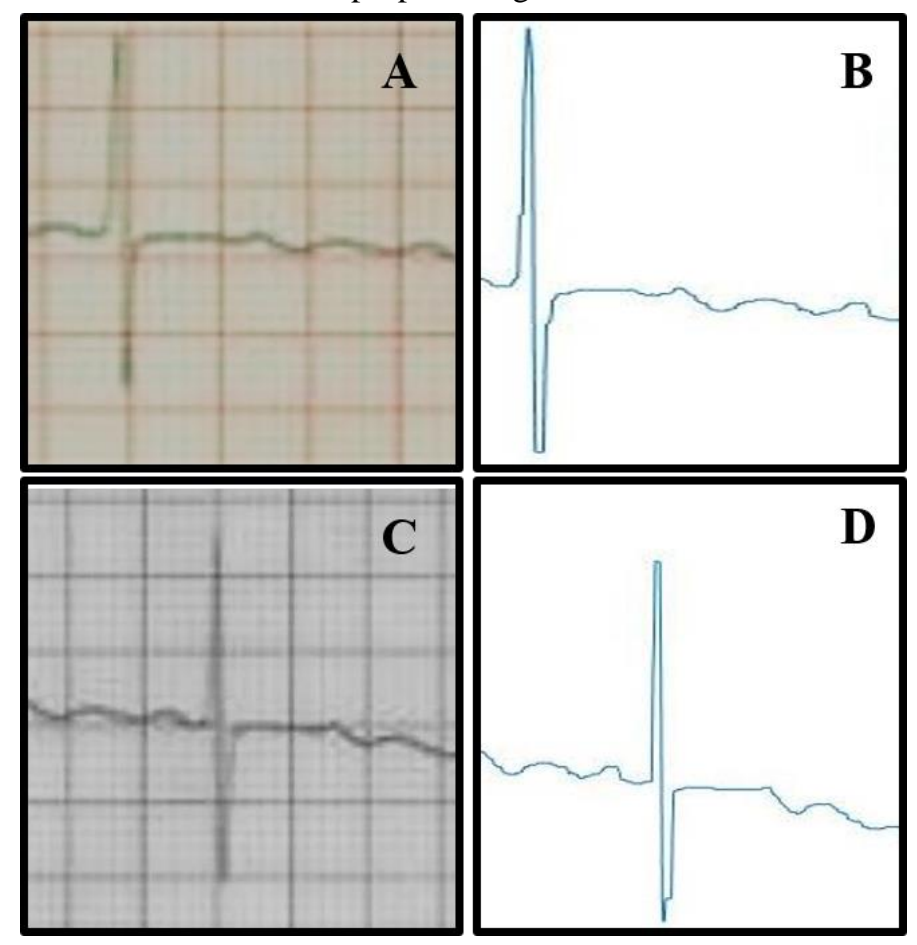

Fig.3. (a) Blurring effect (b) Extracted digitized signal (c) Resolution degradation effect (d) Extracted digitized signal

Blurring effect was found in some images which degrade the visualization with averaging factor $f$. Experimentation was carried out to find the validation of algorithm swapping averaging factors $f$ over entire range and adaptively find the value at which maximum information is achieved. The paper ECG images used for testing are shown in Fig.3(a). The blurring factor was found to be $f=2.5$ and recovered signal is as shown in Fig.3(b). Resolution degradation effect was studied using low resolution scanning. After using k-fill and antialiasing, recovered signal is as shown in Fig.3(d). 

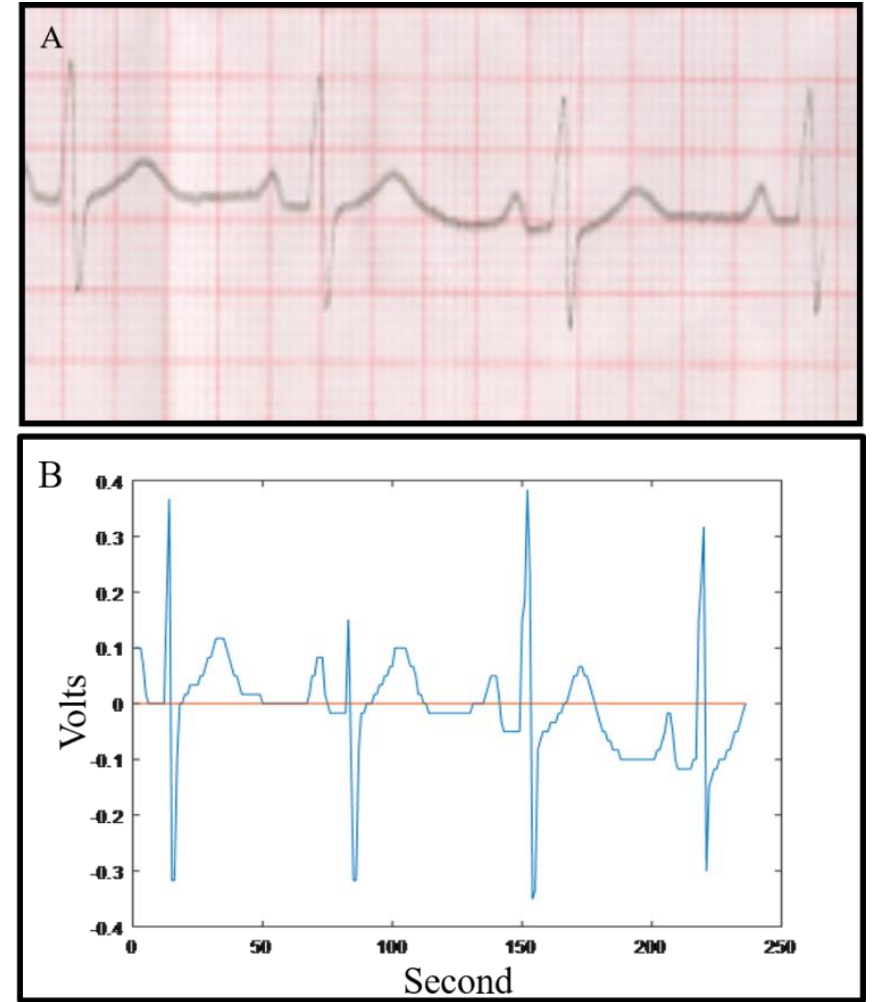

Fig.4. (a) scanned Image (b) Extracted ECG signal with base line

The proposed method does not require any parameter tuning by the user and can deal with degradation occurred due to aging, ink evaporation, folding effect. Extraction of clinically important parameters like HR, R-peak, S-peak and RR-interval and comparing them with pre-diagnosed parameters printed on paper at center top validates the proposed algorithm. The base line or axis detection is done as shown in Fig.4, for extraction of ECG signal parameters.

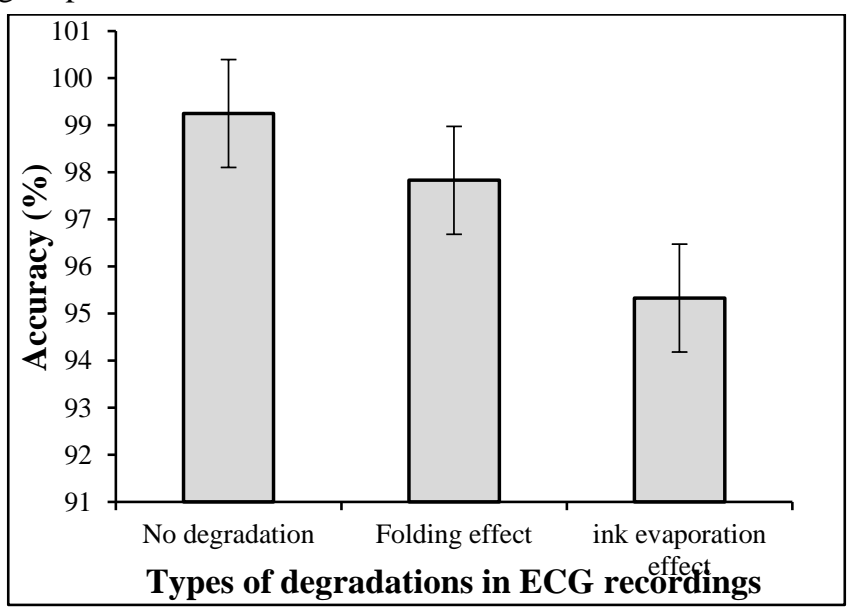

Fig.5. Accuracy achieved after digitizing documented ECG records with different types of degradations

Absolute error $(E)$ is calculated between parameters extracted from digitized signal $\left(P_{d}\right)$ and original paper ECG parameters $\left(P_{o}\right)$, which is used to calculate accuracy of extracted digitized ECG signal.

$$
E=\frac{\left|P_{o}-P_{d}\right|}{P_{o}}
$$

The Fig. 5 shows that as ink evaporates with time it becomes difficult to extract the signals from ECG paper records after scanning them. Hence it is better to scan ECG paper within first few days. As the storage duration increase the accuracy reduces from $99 \%$ to $95 \%$ because of ink evaporation. The Fig.6 shows accuracy is independent of parameter extracted giving average accuracy of $97.33 \%$.

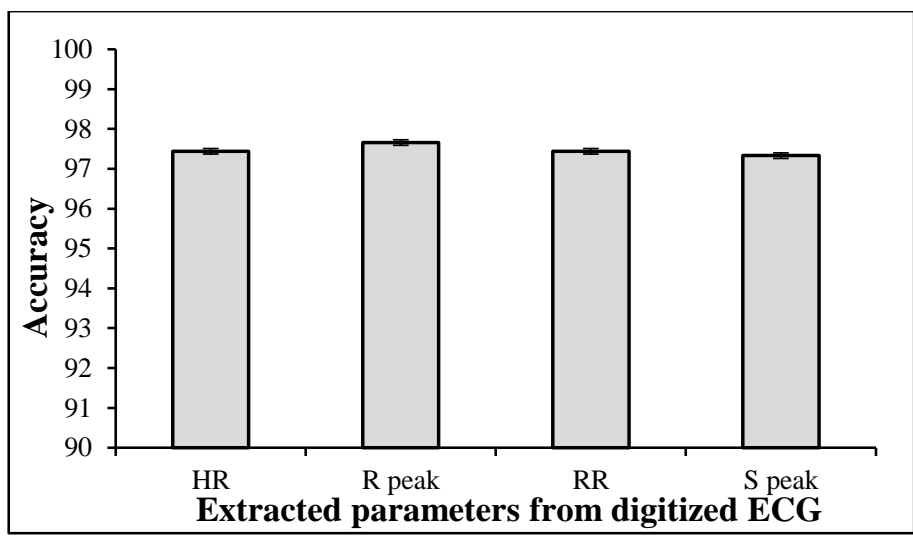

Fig.6. Accuracy of extracted parameters from digitized ECG

Accuracy remains unaffected by type of the parameter extracted.

Table.1. Parametric estimation using PSCM method

\begin{tabular}{|c|c|c|}
\hline $\mathbf{n = 8 3 6}$ & Predicted Yes & Predicted No \\
\hline Actual Yes (716) & $\mathrm{TP}(707)$ & $\mathrm{FN}(09)$ \\
\hline Actual No (120) & $\mathrm{FP}(03)$ & $\mathrm{TN}(117)$ \\
\hline
\end{tabular}

The Table. 1 shows results of PSCM, out of total 836 records 120 are normal cases and remaining 716 were abnormal with problems like sinus bradycardia, sinus tachycardia and atrial flutter. True positive (TP) are correctly predicted abnormal records from 716 abnormal records and false negative $(\mathrm{FN})$ are wrongly predicted abnormal records. Similarly true negative (TN) are correct prediction of normal records and false positive (FP) are false prediction of normal records. PSCM classify total (normal as well as abnormal records) with $98.6 \%$ accuracy.

Table.2. Comparison with other existing systems

\begin{tabular}{|c|c|c|c|c|}
\hline & Accuracy & Specificity & $\begin{array}{c}\text { Error } \\
\text { rate }\end{array}$ & $\begin{array}{c}\text { Sensitivity } \\
\text { (Recall) }\end{array}$ \\
\hline Our algorithm & $98.6 \%$ & $97.5 \%$ & $1.4 \%$ & $98.7 \%$ \\
\hline Chazal et al. [20] & $98.8 \%$ & $81.9 \%$ & $1.2 \%$ & $77.7 \%$ \\
\hline Lu et al. [21] & $89.4 \%$ & $95.0 \%$ & $10.6 \%$ & -- \\
\hline Heden et al. [22] & $90.85 \%$ & $95.4 \%$ & $10.5 \%$ & $84.5 \%$ \\
\hline
\end{tabular}

\section{DISCUSSION}

The results revealed that the signal obtained through digitization process retained the information from the original paper ECG signal. Compared with currently available techniques, 
the key feature of the proposed technique is that it can enable the reconstruction of the original ECG signal despite the degradations that usually occur in archived paper ECG records. The paper ECG degradation effects such as the blurring effect, folding effect, ink evaporation etc. are robustly handled using $\mathrm{k}$ fill and antialiasing method for extraction of ECG signal from degraded ECG paper records.

Scanning is required for converting a 2-D image into a 1-D signal. Many researchers generally prefer horizontal scanning. However, because a signal traverses in the horizontal direction, a vertical scan yields a more detailed signal capture. Moreover, vertical scanning improves the time complexity from $\mathrm{O}(n \log n)$ to $O(n)$ and the space complexity of the proposed digitization method. This indicated that the time and space complexity of vertical scan are improved with respective to the horizontal scan for this application. This algorithm converts the ECG image into digital signals, and it scaled as per the requirement of users/analyzers.

For validating the proposed method, clinically important features of digitized paper ECG signals are extracted. For the blurred ECG samples recorded before 1000 days, average accuracy of extracted parameters from digitized paper ECG record is $95 \%$. The average accuracy for the ECG recordings before 384 days is $98 \%$ and those for current recordings it is $99 \%$, respectively. Hence the average accuracy achieved is $97.33 \%$. The difference between the extracted features from the digitized blurred, folded, and noisy paper ECG records and those on paper ECG records are within the acceptable range as consulted with cardiologist, thus validating the proposed method.

PSCM correctly predicted 824 records out of total 836 paper ECG records. Hence accuracy of PSCM algorithm was $98.6 \%$. Due to broken signal trace, folding effect, low resolution effect nine records are falsely detected as normal and three records are undetected and predicted as abnormal. This is due to aging effect of paper ECG records.

\section{CONLUSIONS}

This paper addresses various degradation problems for paper ECG records, such as the resolution degradation effect, blurring effect, aging effect, folding effect etc. by incorporating the k-fill algorithm and modified anti-aliasing method for ECG signal retrieval. In this study, the proposed method robustly resolves the degradation of 836 ECG paper records recorded for the duration of 0 to 1000 days and improved the time and space complexity through vertical scanning. The accuracy achieved is $97.33 \%$ which is sufficient for correct diagnosis. PSCM correctly predicts 824 records out of total 836 paper ECG records giving prediction accuracy of $98.6 \%$. As the storage duration increase the digitization and diagnosis accuracy reduces because of ink evaporation, aging effect, folding effect etc. Hence it is better to scan and digitize ECG paper record within first few days. The digitized signal obtained from old records can be analyzed and reused in retrospective studies by research organizations. This work can be considered as preliminary expert system at remote places in absence of medical expert.

\section{REFERENCES}

[1] A.D. Jeyarani and T. Jaya Singh, "Analysis of Noise Reduction Techniques on QRS ECG Waveform-by applying Different Filters", Proceedings of IEEE Recent Advances in Space Technology Services and Climate Change, 2010.

[2] Guy A. Story et al., "The Right Pages Image-based Electronic Library for Alerting and Browsing", Computer, Vol. 25, No. 9, pp. 17-26, 1992.

[3] Nucharee Premchaiswadi, Sukanya Yimgnagm and Wichian Premchaiswadi, "A Scheme for Salt and Pepper Noise Reduction and its Application for OCR Systems", WSEAS Transactions on Computers, Vol. 9, No. 4, pp. 351-360, 2010.

[4] Atena Farahmand, Abdolhossein Sarrafzadeh and Jamshid Shanbehzadeh, "Noise Removal and Binarization of Scanned Document Images Using Clustering of Features", Proceedings of International Multi Conference of Engineers and Computer Scientists, Vol. 1, pp. 1-5, 2017.

[5] Atena Farahmand, Abdolhossein Sarrafzadeh and Jamshid Shanbehzadeh, "Document Image Noises and Removal Methods", Proceedings of International Multi Conference of Engineers and Computer Scientists, Vol. 1, pp. 101-105, 2013.

[6] William T. Baxter et al., "Technical Features of a CCD Video Camera System to Record Cardiac Fluorescence Data", Annals of Biomedical Engineering, Vol. 25, No. 4, pp. 713-725, 1997.

[7] Joseph N. Said, Mohamed Cheriet and Ching Y. Suen, "Dynamical Morphological Processing: A Fast Method for Base Line Extraction", Proceedings of $13^{\text {th }}$ International Conference on Pattern Recognition, Vol. 2, pp. 15-20, 1996.

[8] Lei Xu, Erkki Oja and Pekka Kultanen, "A New Curve Detection Method: Randomized Hough Transform (RHT)", Pattern Recognition Letters, Vol. 11, No. 5, pp. 331-338, 1990.

[9] Huaigu Cao, Rohit Prasad and Prem Natarajan, "A Stroke Regeneration method for Cleaning Rule-Lines in Handwritten Document Images", Proceedings of International Workshop on Multilingual OCR, pp. 10-16, 2009.

[10] Zhixin Shi, Srirangaraj Setlur and Venu Govindaraju, "Removing Rule-Lines from Binary Handwritten Arabic Document Images using Directional Local Profile", Proceedings of $20^{\text {th }}$ International Conference on Pattern Recognition, pp. 1916-1919, 2010.

[11] T.W. Shen and T.F. Laio, "Image Processing on ECG Chart for ECG Signal Recovery", Proceedings of IEEE Computers in Cardiology, pp. 20-24, 2009.

[12] Fabio Badilini et al., "ECGScan: A Method for Conversion of Paper Electrocardiographic Printouts to Digital Electrocardiographic Files", Journal of Electrocardiology, Vol. 38, No. 4, pp. 310-318, 2005.

[13] Guojie Shi, Gang Zheng and Min Dai, "ECG Waveform Data Extraction from Paper ECG Recordings by K-means Method", Proceedings of IEEE Computing in Cardiology, pp. 1-5, 2011.

[14] Lakshminarayan Ravichandran et al., "Novel Tool for Complete Digitization of Paper Electrocardiography Data", 
IEEE Journal of Translational Engineering in Health and Medicine, Vol. 1, pp. 1-7, 2013.

[15] W.T. Lawson et al., "New Method for Digitization and Computerized Analysis of Paper Recordings of Standard 12Lead Electrocardiograms", Proceedings of IEEE Computers in Cardiology, pp. 340-347, 1995.

[16] Kirsten Hermes, Tim Brookes and Christopher Hummersone, "The Influence of Dumping Bias on Timbral Clarity Ratings", Audio Engineering Society, pp. 1-139, 2015.

[17] George S.Waits and Elsayed Z. Soliman, "Digitizing Paper Electrocardiograms: Status and Challenges", Journal of Electrocardiology, Vol. 50, No. 1, pp.123-130, 2017.

[18] A. Rajani, "Digitization of Electrocardiography Data Sheet Through Image Processing Techniques", IUP Journal of Electrical and Electronics Engineering, Vol. 9, No. 2, pp. 116-120, 2016.
[19] Fernando Lozano-Fernandez et al., "Auto-Cropping of Phone Camera Color Images to Segment Cardiac Signals in ECG Printouts", Proceedings of IEEE Computing in Cardiology Conference, pp. 1-4, 2016.

[20] Philip de Chazal, M. O'Dwyer and R.B. Reilly, “Automatic Classification of Heartbeats using ECG Morphology and Heartbeat Interval Features", IEEE Transactions on Biomedical Engineering, Vol. 51, No. 7, pp. 1196-1206, 2004.

[21] H. L. Lu, K. Ong and P. Chia, "An Automated ECG Classification System based on a Neuro-Fuzzy System", Proceedings of IEEE Computers in Cardiology, pp. 387390, 2000.

[22] Bo Heden et al., "Acute Myocardial Infarction Detected in the 12-lead ECG by Artificial Neural Networks", Circulation, Vol. 96, No. 6, pp. 1798-1802, 1997. 